\title{
A Consistência das Memórias da Infância ${ }^{1}$
}

\author{
Maria Salomé Pinho ${ }^{2}$ \\ Marta Oliveira \\ António Castro Fonseca \\ Faculdade de Psicologia e de Ciências da Educação \\ Universidade de Coimbra
}

\begin{abstract}
RESUMO - A coleta de relatos retrospetivos constitui um procedimento frequente em psicologia. Neste estudo, analisou-se, em uma amostra de adultos jovens não institucionalizados, a consistência desses relatos sobre diversas experiências da infância (vitimação, medos, dificuldades de aprendizagem), confrontando-os com os relatos atuais dos seus pais e/ou professores. Os resultados mostraram uma relação significativa entre os relatos retrospetivos dos próprios participantes e as informações atuais fornecidas pelas outras fontes, exceto para os medos na infância. A magnitude dessa relação variou entre pequena e média. Com base nesses resultados, sugere-se que, nos estudos que recorram a relatos retrospetivos e atuais,indiquem-se medidas de relação entre relatos, bem como a sua magnitude, de modo a melhor se avaliar a qualidade das inferências baseadas nesses relatos.
\end{abstract}

Palavras-chave: memórias da infância, memória autobiográfica, consistência de recordações, auto-relato

\section{The Consistency of Childhood Memories}

\begin{abstract}
Retrospective self-reports are a common procedure in psychology. The aim of the present study was to analyze, in a large sample of not institutionalized young adults, the consistency of their reports on several childhood experiences (victimization, fears, learning difficulties), by contrasting them with concurrent reports provided by their parents and/or teachers. Results showed a significant relationship between participants` retrospective self-reports and concurrent information provided by other sources, with the exception of childhood fears. The size of that relationship varied between poor and average. Accordingly, it is recommended that studies using retrospective self-reports provide a measure of the relationship between reports, as well as a size measure, so that the quality of inferences based on these reports could be more easily evaluated.
\end{abstract}

Keywords: early memories, autobiographical memory, consistency of memories, self-report

Psicólogos e outros profissionais (e.g., médicos, polícias e juízes) recorrem frequentemente a relatos retrospetivos sobre aspectos mais ou menos recentes da vida passada de um indivíduo. No caso dos psicólogos, esse recurso à memória baseia-se no fato de os traços de aspetos cognitivos e/ou emocionais do passado pessoal terem influência no comportamento atual dos indivíduos (Ross \& Buehler, 1994). Desse modo, averiguar a validade dos relatos retrospetivos, mais ou menos extensos, surge como uma questão essencial (e.g., Schwartz \& Sudman, 1994).

A memória autobiográfica ou memória do passado pessoal é um tipo de memória complexo na sua estrutura e modo de funcionamento (Conway, 2005; Conway \& PleydellPearce, 2000; Wells, Morrisson, \& Conway, 2013). Essa memória compreende duas categorias de informação: o conhecimento de fatos da própria vida (e.g., o nome da escola onde se concluiu o $1^{\circ}$ ciclo), denominado conhecimento autobiográfico, e o conhecimento de aspectos específicos ou detalhes particulares de vivências passadas (e.g.,

1 Trabalho realizado no âmbito do projeto PTDC/PSI-PED/104849/2008, "Comportamento anti-social e problemas de saúde mental em jovens adultos: continuidades e descontinuidades".

2 Endereço para correspondência: Faculdade de Psicologia e de Ciências da Educação, Universidade de Coimbra, Rua do Colégio Novo, Coimbra, Portugal. CEP: 3000-112.E-mail: salome@fpce.uc.pt particularidades da festa de aniversário quando se completou 15 anos), designado por conhecimento episódico ou memórias episódicas. O sentido de identidade proporcionado pela memória não se pode basear apenas em fatos ocorridos, mas depende, também, dos pensamentos, emoções e metas do indivíduo que tornam, assim, a experiência passada significativa (Pasupathi \& Wainryb, 2010). O grau de exatidão da memória autobiográfica pode variar com o tipo de informação recordada. De modo geral, a exatidão será maior quando a informação se refere ao conhecimento da vida do próprio indivíduo em comparação com a informação relativa a acontecimentos particulares ou a episódios que foram pessoalmente vivenciados (British Psychological Society, 2008).

Segundo Brewer (1994), tanto a perspetiva da memória autobiográfica, que coloca a ênfase em uma cópia fotográfica do sucedido em acontecimentos passados, como a perspetiva que dá primazia à reconstrução do vivido não são apoiadas empiricamente. O relato do passado pessoal seria moldado de acordo com as expetativas, estereótipos, crenças e metas atuais do indivíduo (e.g., Neisser, 1994; Pillemer \& White, 1989; Wang, Leichtman, \& White, 1998), que incidem sobre informações gerais registradas sob a forma de esquemas (Renk, Roberts, Klein, Rojas-Vilches, \& Sieger, 2005). Também a forma como os indivíduos lidam com experiências 
passadas emocionalmente negativas poderá influenciar a recordação destas (Bohanek, Fivush, \& Walker, 2005). Contudo, a extensão da influência do que se passa com o indivíduo no momento em que ocorrem as recordações não é consensual. Por exemplo, apesar de se considerar que o estado de humor no momento da recuperação da informação da memória enviesa o relato retrospetivo (Bower, 1981), a distorção provocada poderá ser menor do que se esperaria (Hardt \& Rutter, 2004). No entanto, muitos acontecimentos não são, sequer, registrados de forma duradoura na memória do indivíduo (McLean, Pasupathi, \& Pals, 2007; Westbury \& Dennett, 2000), não podendo, portanto, ser recuperados, enquanto outros são registrados sem os detalhes que poderão ser fundamentais no relato solicitado. Nesse último caso, os indivíduos podem procurar preencher a informação que falta na sua memória com informações obtidas através de inferências ou estimativas, feitas a partir de pressupostos gerais sobre determinados eventos ou, ainda, baseando-se em fontes externas de informação (Loftus, 1975; Loftus \& Cahill, 2007).

Além disso, resultados de estudos empíricos sobre a exatidão das recordações autobiográficas episódicas (conhecimento episódico de situações vividas) mostram a existência de diferenciações nesse tipo de conhecimento (Wells et al., 2013). Assim, para Hardt, Sidor, Bracko e Egle (2006), há experiências vividas na infância que seriam relatadas pelo indivíduo de forma muito confiável (e.g., morte ou divórcio dos pais), outras que seriam recordadas com um grau médio de confiabilidade (e.g., situações de abuso) e outras que apresentariam pouca confiabilidade (e.g., um membro da família ter estado na prisão). Segundo os mesmos autores, porém, também não há consenso entre os resultados dos estudos, pois os relatos retrospetivos sobre um determinado tipo de vivência pessoal podem apresentar baixa confiabilidade em alguns estudos e maior confiabilidade em outros.

Várias razões, principalmente de natureza metodológica, poderão ser invocadas para explicar essas discrepâncias. Primeiro, a maneira como são formulados os itens/questões incluídos nos instrumentos utilizados na coleta das memórias pessoais - por exemplo, a clareza dos itens ou a ocorrência de desejabilidade social - poderá afetar a qualidade dos relatos retrospetivos obtidos (Hardt et al., 2006). Segundo, há de se considerar aspectos que se prendem com as diferenças sexuais. Apesar de se tratar de um assunto com poucos estudos publicados, de modo geral, considera-se que as mulheres fazem relatos retrospetivos de memórias da sua vida pessoal mais detalhados (McLean et al., 2007) e mais exatos. Essa superioridade das mulheres diz respeito, sobretudo, a determinados aspectos dos episódios experienciados, mais concretamente àqueles cuja recordação seria favorecida pela maior atenção dada a características emocionais (Grysman \& Hudson, 2013). No entanto, a idade do indivíduo poderá ter também um papel importante, uma vez que, em adultos mais novos, comparativamente com adultos mais velhos (57-80 anos), a referida diferença entre mulheres e homens quanto ao relato de memórias episódicas pessoais poderá não ser observada (Ros \& Latorre, 2010).Daí que, terceiro, fatores relativos à idade dos indivíduos mostram-se ainda relevantes. Em suma, o conhecimento autobiográfico que diz respeito a fatos da vida pessoal pode ser pouco exato, envolvendo, por exemplo, incorreção na indicação da idade em que um indivíduo diz ter começado a consumir substâncias ilícitas (Ensminger, Juon, \& Green, 2007).

Uma maneira de superar esses problemas seria considerar a convergência entre várias medidas e fontes de informação (e.g., Offer, Kaiz, Howard, \& Bennett, 2000). Porém, como sublinham Ensminger et al. (2007), consistência não é o mesmo que validade, constituindo aquela um aspeto desta. A validade significa robustez dos resultados ou das medidas e que estes expressam com exatidão o que se passa na realidade (Sullivan, 2011). A confiabilidade, que resulta da consistência, distingue-se da validade. Note-se que o relato de um mesmo indivíduo ao longo do tempo pode ser consistente, mas incorreto, i.e., não válido (White, Widom, \& Chen, 2007) e o inverso também pode suceder. Nessa última situação, há a considerar as chamadas experiências "escondidas" (e.g., abuso sexual por um membro da família), que, frequentemente, não são mencionadas na altura em que ocorrem, quando o sujeito ainda está na infância (Hardt \& Rutter, 2004).

No que diz respeito aos relatos retrospectivos de acontecimentos pessoais ocorridos na infância, apresentados por outros informantes (e.g., pais) que conviveram com o indivíduo durante esse período de vida, Fisher, Bunn, Jaccobs, Moran e Bifulco (2011) colocaram a hipótese de o relato das mães fornecer informação do mesmo tipo da que é dada pelos próprios filhos. Nesse estudo, que envolveu 160 mães (idade média de 45 anos) com risco elevado para perturbações afetivas e seus respetivos filhos (idade média de 20 anos), analisaram-se as recordações de acontecimentos adversos surgidos antes dos 17 anos de idade. Os resultados mostraram algumas diferenças entre os relatos feitos pelas duas fontes de informação, já que as mães tendiam a relatar menos acontecimentos negativos do que o próprio indivíduo. Os relatos retrospectivos sobre acontecimentos da vida dos seus filhos podem ser distorcidos tornando-se mais positivos, i.e., passando a conformar-se melhor àquilo que é considerado desejável (Coolidge, Tambone, Durham, \& Segal, 2011; Greenhoot, 2011). Em um estudo de Bernard, Killworth, Kronenfeld e Sailor (1984), os relatos retrospetivos feitos pelas mães, por exemplo, sobre as aptidões escolares e o estado de saúde dos filhos continham incorreções, sobretudo devidas a omissões, que atingiam valores entre 35 a 75\%. Em outro estudo, Renk et al. (2005) avaliaram 185 estudantes universitários (com idades compreendidas entre os 18 e os 22 anos) e seus respetivos pais e mães, utilizando o Youth Self-Report (Achenbach, 1991a) e o Child Behavior Checklist (Achenbach, 1991b). Esses autores obtiveram uma correspondência moderada entre os problemas de internalização e de externalização relatados por pais e filhos retrospetivamente. Uma das explicações avançadas pelos autores é a de que os jovens podem ter integrado os relatos históricos dos pais sobre a sua infância e, quando da evocação desses acontecimentos, essa informação passou a fazer parte da história percebida pelo próprio indivíduo.

Por sua vez, Brown (2012), em um trabalho que utilizou dados do estudo longitudinal National Child Development Study, considerou, além dos relatos retrospetivos dos próprios indivíduos aos 50 anos sobre o número de pessoas que 
vivia na sua casa e o número de divisões dessa casa quando tinha 11 anos de idade, os relatos atuais feitos pelos pais (sobretudo, as mães) desses indivíduos. Os seus resultados indicaram que indivíduos já com 50 anos eram capazes de recordar aspetos da sua infância com um razoável grau de exatidão. No grupo dos que foram incapazes de recordar corretamente o que sucedeu na infância, as diferenças entre as respostas atual e retrospetiva foi geralmente pequena. $\mathrm{O}$ autor identificou, ainda, algumas características comuns aos indivíduos que tinham maior probabilidade de fornecer respostas inconsistentes. Estes vinham de famílias instáveis, tinham menos escolaridade ou eram do sexo masculino. Os seus dados revelaram ainda que os indivíduos com maior pontuação em um teste de avaliação da memória (tarefa de evocação de uma lista de palavras) tinham maior probabilidade de fornecer respostas consistentes e, no caso de a resposta não estar correta, a magnitude do erro desses indivíduos seria geralmente inferior, por comparação com a dos indivíduos com menor desempenho mnemônico.Brown (2012) referiu também vários fatores que influenciam os eventos que um indivíduo é capaz de recordar e a forma como o faz. Entre estes, destacou os seguintes: a saliência da informação, a telescopagem anterógrada e retrógrada e o princípio da acessibilidade. De acordo com o fenômeno de telescopagem, o indivíduo assume que o evento ocorreu há muito pouco tempo ou então há muito tempo atrás, relativamente ao tempo certo (Bernard et al., 1984). Segundo o princípio da acessibilidade, eventos que o indivíduo consegue recordar mais facilmente são percebidos como tendo ocorrido mais recentemente e os eventos mais difíceis de recordar são tidos como tendo ocorrido há mais tempo.

Utilizando dados de uma investigação longitudinal portuguesa, o presente estudo analisa a consistência das recordações que jovens adultos (com idades compreendidas entre 25 e 33 anos) têm da ocorrência de determinadas experiências da sua infância. Para tal, comparam-se as respostas dadas por indivíduos jovens a algumas questões específicas sobre eventos ocorridos há cerca de 20 anos (relatos retrospetivos) com a informação que foi recolhida junto dos pais e/ou professores na altura de ocorrência dos eventos (relatos atuais), analisando-se a consistência dessas respostas. Essas questões remetem a aspetos das seguintes situações da infância: vitimação, medos e dificuldades de aprendizagem. Por vitimação, designa-se, habitualmente, um vasto leque de experiências penosas a que criança se viu exposta, cujas consequências negativas podem perdurar até a idade adulta (MacDougall \& Villancourt, 2015). Em particular, neste estudo, foi considerada como um processo de marginalização exercido por outrem investido de poder, sem ocorrer necessariamente violência física (cf., Azevedo \& Guerra, 1989). Os medos na infância dizem respeito ao sentimento de receio de fazer algo ou à reação ansiosa, decorrente da presença de um estímulo físico ou mental, associado a um estado de alerta do organismo (e.g., Murphy, 1985). Trata-se, portanto, de reações naturais a uma ameaça real ou imaginária (Fonseca \& Perrin, 2011). Foi aqui restringido o âmbito do conceito de dificuldades de aprendizagem ao processo de escolarização. Nesse contexto, referem-se a problemas na captação e assimilação de informação que impedem o indivíduo de adquirir os conhecimentos escolares esperados para a idade ou de seguir um percurso acadêmico esperado em seu meio (Rebelo, Fonseca, Simões, \& Ferreira, 1995). Uma vez que se compararam relatos retrospectivos com relatos atuais, como sucedeu no estudo de Brown (2012), é esperado obter uma consistência, pelo menos, razoável entre ambos, já que que os relatos retrospetivos são feitos por sujeitos mais novos do que os participantes do referido estudo. Como é conhecido, a recordação autobiográfica diminui com o aumento do tempo decorrido após o evento em causa (Wagenaar, 1986). Quanto à variável sexo dos sujeitos dos relatos retrospetivos, por se tratar de uma questão com poucos estudos e os seus resultados não serem convergentes, é aqui considerada de forma apenas exploratória. Assim, não se formulou uma hipótese prévia relativamente à influência dessa variável.

O esclarecimento da questão sobre a confiança que se poderá ter no relato de um jovem adulto relativamente a acontecimentos da sua infância é importante, tanto do ponto de vista teórico (e.g., qual a validade da informação obtida em investigações retrospetivas) como do ponto de vista prático, em contexto forense (e.g., qual a validade do testemunho de um adulto sobre acontecimentos da sua infância) e em contexto clínico (e.g., qual a validade do relato de acontecimentos traumáticos e/ou de problemas de saúde mental ocorridos na infância).

\section{Método}

\section{Participantes}

Este estudo faz parte de uma investigação longitudinal, que envolveu uma ampla amostra de rapazes e moças que, no ano letivo 1992-1993, frequentavam o $2^{\circ}, 4^{\circ}$ e $6^{\circ}$ anos de escolaridade em escolas primárias do Concelho de Coimbra. Os seus pais e/ou professores também responderam a perguntas sobre os sujeitos que participaram nesta investigação. Essa investigação compreendeu cinco momentos de avaliação: quando os sujeitos tinham 7-8 anos, 11-12 anos, 14-15 anos, 17-18 anos e, finalmente, 26-27 anos. No presente estudo, apenas foram utilizados dados da coorte mais nova de sujeitos ( $2^{\circ}$ ano de escolaridade). Mais especificamente, analisaram-se dados coletados no $5^{\circ}$ momento, ou seja, em 2012, quando os sujeitos tinham em média 26,56 anos $(D P=1,13$, amplitude $=25-33)$. Os dados relativos aos seus pais e/ou professores referem-se ao primeiro momento de avaliação, i.e., a 1992, quando os seus filhos e/ou alunos tinham 7-8 anos de idade $(M=$ $7,53, D P=, 82$, amplitude $=6-13$ ). Embora no primeiro momento tenham sido coletados dados de 445 sujeitos da coorte mais nova, ao longo do tempo, e devido à mortalidade experimental, o número de sujeitos foi diminuindo, sendo que à última avaliação responderam cerca de 383 sujeitos. Para a coleta de dados, os investigadores deslocaram-se às escolas, residências e outros locais da conveniência dos sujeitos. Previamente à coleta de informação, foi obtido o consentimento escrito dos pais e professores, no primeiro 
momento de avaliação e a dos próprios sujeitos, nos momentos seguintes.

\section{Instrumentos}

Para a coleta de dados, ao longo das sucessivas fases desta investigação, utilizou-se uma variedade de instrumentos (entrevista, ficha de informação geral e inventários) destinada a avaliar diversos domínios da vida dos sujeitos. Particularmente relevantes para as análises deste estudo são o Inventário de Problemas do Comportamento para Pais (Child Behaviour Checklist [CBCL]; Achenbach, 1991b; versão portuguesa de Fonseca, Simões, Rebelo, Ferreira, \& Cardoso, 1994) e o Inventário de Comportamento da Criança para Professores (Teacher Report Form [TRF]; Achenbach, 1991c; versão portuguesa de Fonseca, Simões, Rebelo, Ferreira, \& Cardoso, 1995).

O CBCL é composto por 113 itens abrangendo uma grande diversidade de problemas de comportamento da criança e do adolescente. Cada item pode ter uma pontuação de 0 (nunca verdade), 1 (às vezes verdade) e 2 (sempre verdade), referindo-se ao período dos últimos 6 meses. Os itens estão distribuídos por oito escalas: Comportamento Agressivo, Delinquência, Ansiedade/Depressão, Problemas de Pensamento, Problemas de Atenção, Queixas Somáticas, Isolamento e Problemas Sociais. Algumas dessas escalas podem ser agrupadas, de maneira a originar uma escala ou cluster de problemas de externalização (externalizing problems) e outra de problemas emocionais ou de internalização (internalizing problems).

O TRF apresenta os mesmos conteúdos, estrutura e modo de resposta do CBCL já descrito, mas é preenchido pelos professores. O CBCL e o TRF foram anteriormente objeto de um estudo de adaptação e de estandardização para a população portuguesa (Albuquerque, Fonseca, Simões, Pereira, Rebelo, \& Temudo, 1998; Albuquerque, Fonseca, Simões, Pereira, \& Rebelo, 1999; respetivamente), tendo ambos, de modo geral, revelado boas propriedades psicométricas, embora a estrutura fatorial encontrada na população portuguesa nem sempre coincida com a estrutura obtida na população americana.

\section{Procedimento}

Os sujeitos da primeira coorte (inicialmente no $2^{\circ}$ ano de escolaridade) foram seguidos desde a infância até à idade adulta, tendo sido recolhidas diversas informações dos próprios, dos pais e dos professores sobre vários aspectos do comportamento dos sujeitos. $\mathrm{Na}$ última fase do estudo longitudinal, foram recolhidas informações apenas dos próprios participantes sobre esses mesmos comportamentos ou experiências da sua infância, nomeadamente aspectos como a vitimação, os medos e as dificuldades de aprendizagem na escola.

Mais concretamente, para se analisar a recordação de situações de vitimação (ver Tabela 1) utilizou-se a informação fornecida pelos sujeitos no momento da última avaliação no que se refere às suas experiências de vitimação na infância, comparando-a com a resposta dada pelos pais a uma pergunta semelhante incluída no CBCL (Achenbach, 1991b), por eles preenchido na primeira avaliação. Os sujeitos (já adultos) responderam à pergunta relativa à sua infância Fizeram pouco de si ou chamaram-lhe nomes feios? enquanto os pais tinham respondido anteriormente (quando os filhos tinham 7-8 anos) ao item: Fazem pouco dele(a) frequentemente?

No que tange à recordação da existência ou não de medos na infância (ver Tabela 1), foi colocada aos sujeitos adultos a pergunta Lembra-se de, na infância, ter muito medo de alguma coisa, pessoa ou situação?, cuja resposta seria sim ou não. Essa informação havia sido solicitada aos pais e professores, através do preenchimento do CBCL e do TRF (respectivamente Achenbach, 1991b, 1991c), quando os sujeitos eram ainda crianças (com 7-8 anos). Dos vários itens desses instrumentos, três incidiam sobre os medos da criança, a saber: Tem medo de animais, situações ou lugares (sem ser medo da escola); Tem medo de ir para a escola; Tem medo de poder pensar ou fazer qualquer coisa de mal. As respostas a esses três itens (em uma escala Likert na qual os pais ou professores teriam que assinalar não verdadeira, às vezes verdadeira ou muitas vezes verdadeira) foram somadas para constituir uma variável global de medos referidos pelos pais e outra de medos referidos pelos professores. Assim, a existência de medos na infância referidos por pais ou professores era indicada por uma pontuação superior a 0 nessa variável global, que depois foi comparada com a resposta dos sujeitos na idade adulta.

Relativamente à sua própria memória de dificuldades de aprendizagem (ver Tabela 1) quando da frequência à escola (Na escola, aprendia sempre bem ou acha que tinha dificuldades de aprendizagem?, se respondesse apenas sim ou não, era interrogado para esclarecer o sentido da resposta), as respostas dos sujeitos foram comparadas com as informações prestadas por professores e por pais na primeira avaliação. Nesse momento de avaliação, os seus pais tinham respondido à pergunta $O / A$ seu/sua filho/a aprende bem na escola?; e os seus professores, Este/a aluno/a aprende bem na escola?, perguntas que constava da ficha de informação geral, anexada aos inventários de Achenbach para pais e para professores.

\section{Resultados}

Para a análise estatística dos dados,recorreu-se ao Statistical Package for the Social Sciences (SPSS) versão 22 para Windows (IBM, New York, USA), tendo sido calculado o teste de independência do Qui-quadrado $\left(\chi^{2}\right)$ e, para medir o tamanho do efeito, utilizaram-se o coeficiente Phi $(\varnothing) \mathrm{e}$ o odds ratio $(O R)$. O valor de significância estatística $(p)$ foi fixado em 0,05 . As análises que a seguir se apresentam dizem respeito às recordações dos sujeitos sobre a ocorrência de situações de vitimação, de medos e de dificuldades de aprendizagem na escola, em relação às quais havia já informação fornecida pelos seus pais e/ou professores, quando do primeiro momento de avaliação. Os resultados das respostas dadas pelos próprios, pelos seus pais e professores, encontram-se na Tabela 1. 


\section{Vitimação na Infância}

$\mathrm{Na}$ Tabela 1, pode verificar-se que a ocorrência de vitimação é referida por $21,7 \%$ dos pais (quando os seus filhos tinham 7-8 anos de idade) e por 39,2\% dos próprios sujeitos na idade adulta (referindo-se à sua infância). Com base no resultado do teste $\chi^{2}$,verificou-se que havia independência entre mulheres e homens jovens $\left[\chi^{2}(1, n=380)=0,00, p\right.$ $=0,995]$ no que diz respeito à recordação da vitimação, pelo que, nas análises subsequentes, foi considerada separadamente a subamostra feminina e a subamostra masculina. No grupo das mulheres, foi registrada uma relação estatisticamente significativa entre a sua recordação e a resposta dos pais: $\chi^{2}(1, n=332)=3,88, p=0,049, \varnothing(1$, $167)=0,15, p=0,049, O R=1,54,95 \% I C[1,03,2,31] . \mathrm{Em}$ outras palavras, há uma relação entre a recordação que as mulheres jovens têm da ocorrênciade vitimações na infância e o relato atual dessas vitimações feito pelos seus pais, no momento da primeira avaliação.Esse efeito apresenta um valor considerado pequeno. As chances de as mulheres jovens recordarem ter vivido situações de vitimação na sua infância, tendo os seus pais relatado a sua ocorrência, foram 1,54 vezes maiselevadas do que quando as mulheres não recordaram ter sido alvo de vitimação. A confiança para o nível 95\% desse odds ratio expressar a maior tendência de as mulheres jovens recordarem experiências de vitimação da sua infância, relativamente a não recordarem tal situação quando os seus pais relataram contemporaneamente essa ocorrência, varia entre 1,03 e 2,31. No que diz respeito à subamostra masculina, não foi alcançado o limiar de significância

Tabela 1. Percentagem de respostas sim para os itens do CBCL, TRF e ficha de informação geral

\begin{tabular}{|c|c|c|c|c|}
\hline Experiências da infância & Itens / perguntas & $\begin{array}{c}\text { Pais } \\
\%\end{array}$ & $\begin{array}{c}\text { Professores } \\
\% \\
\end{array}$ & $\begin{array}{c}\text { Próprio } \\
\%\end{array}$ \\
\hline \multicolumn{5}{|l|}{ Vitimação (n = 380) } \\
\hline Infância & $\begin{array}{l}\text { Fazem pouco dele/a } \\
\text { frequentemente(CBCL) }\end{array}$ & 21,7 & & \\
\hline Adultez & $\begin{array}{l}\text { Fizeram pouco de si ou } \\
\text { chamaram-lhe nomes feios (Ficha } \\
\text { de informação geral) }\end{array}$ & & & $\begin{array}{l}39,2(\mathrm{M}) \\
39,2(\mathrm{H})\end{array}$ \\
\hline \multicolumn{5}{|l|}{$\operatorname{Medos}(\mathrm{n}=382)$} \\
\hline & $\begin{array}{l}\text { Tem medo de animais, situações, } \\
\text { lugares; de ir para a escola; de } \\
\text { poder pensar ou fazer qualquer } \\
\text { coisa de mal (CBCL) }\end{array}$ & 63,2 & & \\
\hline Infância & $\begin{array}{l}\text { Tem medo de animais, situações, } \\
\text { lugares; de ir para a escola; de } \\
\text { poder pensar ou fazer qualquer } \\
\text { coisa de mal (TRF) }\end{array}$ & & 28,6 & \\
\hline Adultez & $\begin{array}{l}\text { Lembra-se de, na infância, ter } \\
\text { muito medo de alguma coisa, } \\
\text { pessoa ou situação? (Ficha de } \\
\text { informação geral) }\end{array}$ & & & 31,2 \\
\hline \multicolumn{5}{|l|}{$\begin{array}{l}\text { Dificuldades de } \\
\text { Aprendizagem }(n=383)\end{array}$} \\
\hline & $\begin{array}{l}\mathrm{O} / \mathrm{A} \text { seu/sua filho/a aprende bem } \\
\text { na escola?(Ficha de informação } \\
\text { geral) }\end{array}$ & 28,1 & & \\
\hline Infância & $\begin{array}{l}\text { Este/a aluno/a aprende bem na } \\
\text { escola?(Ficha de informação } \\
\text { geral) }\end{array}$ & & 38,5 & \\
\hline Adultez & $\begin{array}{l}\text { Na escola, aprendia sempre } \\
\text { bemou acha que tinha } \\
\text { dificuldades de aprendizagem? } \\
\text { (Ficha de informação geral) }\end{array}$ & & & 40,7 \\
\hline
\end{tabular}

Nota . CBCL = Child Behaviour Checklist (Achenbach, 1991b; versão portuguesa de Fonseca et al., 1994), TRF = Teacher Report Form (Achenbach, 
estatística, cruzando a informação recordada pelos próprios na idade adulta com a informação atual referida pelos seus pais $\left[\chi^{2}(1, n=165)=0,84, p=0,361\right]$. O que quer dizer que a informação proveniente dos relatos retrospectivos e dos relatos atuais não se encontra relacionada.

\section{Medos da Infância}

Por terem sido encontradas diferenças significativas entre as mulheres e os homens $\left[\chi^{2}(1, n=382)=9,08, p=0,003\right]$ quanto aos seus relatos retrospetivos sobre os medos na infância, i.e., não haver independência entre a recordação de mulheres e de homens sobre essa situação, não se procedeu à diferenciação da amostra quanto ao sexo. Como indicado na Tabela $1,31,2 \%$ dos sujeitos adultos recordaram ter tido medos na sua infância enquanto $63,2 \%$ dos seus pais e $26,8 \%$ dos seus professores, referiram essa situação. Sobre os medos na infância, não foi encontrada relação entre a resposta dos pais e a recordação dos próprios sujeitos $\left[\chi^{2}(1, n=382)=\right.$ $1,58, p=0,209]$ nem entre a recordação destes e o relato atual dos seus professores $\left[\chi^{2}(1, n=382)=0,26, p=0,608\right]$. Portanto, a recordação que os sujeitos têm, na idade adulta, dos medos que sentiam na infância não foi confirmada pela informação dada pelos pais e professores nessa altura.

\section{Dificuldades de Aprendizagem na Escola}

Quanto à análise dos relatos retrospetivos sobre as dificuldades de aprendizagem na escola, começou-se, igualmente, por calcular o teste $\chi^{2}$ atendendo ao sexo dos sujeitos. Os resultados obtidos apontam, novamente, para a rejeição da independência entre a recordação de mulheres e de homens $\left[\chi^{2}(1, n=383)=12,14, p<0,001, \varnothing(1,383)=0,18\right.$, $p<0,001]$, tomando-se, então, a amostra conjunta nas análises seguintes. Na Tabela 1, pode observar-se que a existência de dificuldades de aprendizagem na escola é mencionada por $40,7 \%$ dos próprios sujeitos na idade adulta (referindo-se à sua infância), por $28,1 \%$ dos pais e $38,5 \%$ dos professores (quando os seus filhos e alunos, respectivamente, tinham 7-8 anos de idade). A comparação entre os relatos retrospetivos sobre a existência de dificuldades de aprendizagem e os relatos atuais dos pais revelou-se significativa $\left[\chi^{2}(1, n=331)\right.$ $=54,85, p<0,001]$, de magnitude média: $\varnothing(1,331)=0,41$, $p<0,001$ e $O R=2,68,95 \%$ IC $[2.09,3.43]$. Nesse caso, as chances de os adultos jovens recordarem dificuldades de aprendizagem na escola, quando os seus pais relataram a ocorrência dessas dificuldades, foram 2,68 vezes mais elevadas do que quando os adultos jovens não recordaram essa situação. A confiança para o nível $95 \%$ desse odds ratio mostrar a maior tendência de os adultos jovens retrospetivamente relatarem ter vivenciado dificuldades de aprendizagem na escola, comparativamente a não recordarem tal ocorrência, quando os seus pais mencionaram na altura essa situação, varia entre 2,09 e 3,43. Aliás, o mesmo sucedeu na comparação das recordações dos sujeitos com os relatos atuais dos professores $\left\{\chi^{2}(1, n=368)=54,37, p<0,001\right.$, $\varnothing(1,368)=0,38, p<0,001, O R=2,47,95 \%$ IC $[1,93,3,15]\}$ relativamente à ocorrência de dificuldades de aprendizagem.
O tamanho do efeito foi de valor médio. As chances de os adultos jovens recordarem dificuldades de aprendizagem na escola, tendo os seus professores indicado a ocorrência dessas dificuldades foram 2,47 vezes mais elevadas do que quando os adultos jovens não recordaram essa situação. A confiança para o nível $95 \%$ desse odds ratio indicar a maior tendência de os adultos jovens que recordaram ter vivenciado dificuldades de aprendizagem na escola, relativamente a não recordarem tal ocorrência, quando os seus professores relataram essa situação, varia entre 1,93 e 3,15. Em suma, observou-se uma associação moderada entre a recordação dos próprios sujeitos, cerca de 20 anos mais tarde, sobre a existência de dificuldades de aprendizagem na escola e o relato de outras fontes de informação (pais e professores) nessa altura.

\section{Discussão}

O objetivo deste trabalho consistiu em examinar em que medida as recordações que os adultos jovens têm da sua infância são confiáveis, considerando a sua consistência ou relação com relatos atuais dos pais e/ou professores. Para tal, confrontamos os relatos que, aos 26-27 anos de idade, os sujeitos fazem da ocorrência de algumas situações da sua infância (vitimação, medos e dificuldades de aprendizagem na escola) com os relatos dos pais e professores quando os sujeitos tinham 7-8 anos de idade. Os resultados obtidos apontam para a existência de relação entre relatos retrospetivos dos adultos jovens e relatos atuais provenientes de outras fontes, como os seus pais e professores, exceto no que diz respeito a medos tidos na infância. Assim, para a ocorrência de experiências de vitimação e de dificuldades de aprendizagem na infância, a relação entre a recordação dos próprios sujeitos, cerca de 20 anos mais tarde (mulheres jovens e adultos jovens, respectivamente) e a informação fornecida pelos seus pais e/ou professores na altura de ocorrência dessas situações variou entre fraca e média.

Considerados no seu conjunto, os resultados do presente estudo são concordantes com os obtidos por Brown (2012), embora o tipo de informação solicitado nos relatos assim como a idade em que foram coletados os relatos retrospetivos sobre situações pessoais da infância sejam diferentes em ambos os estudos. Os sujeitos no estudo de Brown (2012) responderam a perguntas sobre a casa de infância (número de divisões e de pessoas que viviam nessa casa) e os relatos retrospetivos foram coletados quando os sujeitos já se encontravam na meia-idade.

Por sua vez, no que diz respeito aos relatos sobre dificuldades de aprendizagem na escola, a magnitude da relação encontrada foi superior à mencionada por Bernard et al. (1984). Há, no entanto, algumas diferenças relevantes entre os dois estudos,a saber: no caso do estudo de Bernard et al. (1984), a utilização de relatos retrospetivos das mães (relativamente aos quais também se levantam problemas de confiabilidade; e,g., Coolidge et al., 2011) e o tipo de perguntas colocadas (as aptidões escolares).

As diferenças das respostas quanto à variável sexo nos relatos retrospetivos de situações de vitimação na infância poderão explicar-se, pelo menos em parte, com o maior 
rigor e detalhe das mulheres nos relatos de memórias da vida pessoal, principalmente quando estão envolvidas caraterísticas de natureza emocional (Grysman \& Hudson, 2013). Porém, como foi referido, os resultados dos estudos não são consensuais a este respeito (e.g., Ros \& Latorre, 2010). A propósito da dimensão dessa relação ser fraca, entre o relato retrospetivo de mulheres jovens e o relato dos seus pais, Hardt et al. (2006) mencionam as situações de abuso entre as experiências da infância relatadas retrospetivamente com grau médio de confiabilidade. As experiências de vitimação contempladas no presente estudo não configuram propriamente situações de abuso e, apesar de se tratar também de situações com carga emocional, a sua recordação na vida adulta, cerca de 20 anos mais tarde, poderá ser menor, devido à diminuição da sua intensidade emocional (cf., Ritchie et al., 2014).

A não observação de acordo entre a recordação de medos na infância e a informação dada a esse respeito pelos pais e professores poderá estar relacionada com o fato de determinados medos das crianças terem um caráter normativo e serem superados pelo sujeito naturalmente, seguindo um padrão adaptativo de desenvolvimento (Beesdo, Knappe, \& Pine, 2009). Por outro lado, os seus pais e professores identificaram esses medos na altura, independentemente do seu caráter normativo. De modo geral, é ainda de notar que a forma como os indivíduos lidam com experiências passadas emocionalmente negativas (Bohanek et al., 2004), as suas expectativas, estereótipos, crenças e metas atuais (Neisser, 1994; Pillemer \& White, 1989; Wang et al., 1998) poderão influenciar a recordação dessas experiências. Há também a assinalar, relativamente a essa questão dos medos na infância, um problema de validade interna, que se relaciona com a formulação dos itens no CBCL e no TRF ser mais específica e abrangente do que a pergunta sobre os medos, inserida na ficha de informação geral, à qual responderam os adultos jovens. Ora, sabe-se que os conteúdos veiculados pelos itens, assim como a sua clareza, constituem aspectos importantes que condicionam os relatos dos sujeitos (Hardt et al., 2006), afetando, portanto, a comparação das respostas obtidas.

Importa ainda notar que os relatos de outros informantes, embora seja um procedimento recomendado para aumentar a validade dos relatos retrospetivos (Offer et al., 2000), levantam o problema da comparabilidade (Hardt \& Rutter, 2004) e introduzem fontes de variação adicional (e.g., experiências de vida pessoal podem ser diferentemente valorizadas e objeto de significação diversa pelo próprio e por outrem). A recolha de relatos atuais dos próprios sujeitos, não contemplada no presente estudo, poderia também fornecer informação relevante, dado que se incidiu sobre aspetos gerais de experiências vividas na infância (ocorrência ou não de uma determinada situação). Em contextos em que se consideram os conteúdos específicos dessas vivências, o seu relato detalhado pode ser difícil para as crianças, devido a caraterísticas do desenvolvimento da memória autobiográfica (Picard, Eustache, \& Piolino, 2009). Por exemplo, antes dos 5-7 anos, não estariam presentes caraterísticas essenciais da memória autobiográfica, como a recordação consciente específica, i.e., com detalhes espaço-temporais e sensoriais.

Independentemente dos resultados dos estudos realizados, os relatos retrospetivos têm-se mantido como uma ferramenta comumente utilizada em diversas áreas da psicologia, quer em domínios básicos quer aplicados. Perante a variabilidade dos resultados encontrados na literatura, além das precauções relativas ao método/procedimentos adotados na coleta dos relatos, apontadas anteriormente e que não são propriamente específicas deste modo de inquirir, o recurso a relatos retrospetivos e atuais deverá ser acompanhado de medidas de relação ou concordância e ser sempre indicada a sua magnitude. Cremos que esse procedimento irá contribuir para uma avaliação mais eficaz da qualidade das inferências com base em relatos retrospetivos. Nesse sentido, seria ainda útil analisar, em futuros trabalhos, o efeito das diferenças individuais e sociais sobre a consistência da informação atual (por comparação com a informação recolhida na infância) dada pelo sujeito (nomeadamente o resultado da avaliação da memória deste), para desse modo se poder validar, com maior ou menor certeza, as informações relatadas.

\section{Referências}

Achenbach, T. M. (1991a). Manual for the Youth Self-Report and 1991 Profile. Burlington, V.T.: University of Vermont, Department of Psychiatry.

Achenbach, T. M. (1991b). Manual for the Child Behavior Checklist/4-18 and 1991 Profile. Burlington, V.T.: University of Vermont, Department of Psychiatry.

Achenbach, T. M. (1991c). Manual for the Teacher Report Form and 1991 Profile. Burlington, V.T.: University of Vermont, Department of Psychiatry.

Albuquerque, C. P., Fonseca, A. C., Simões, M. R., Pereira, M., \& Rebelo, J. A. (1999). O Inventário de Comportamento da Criança para Professores: Estudo com uma amostra clínica. Psychologica, 21, 113-128.

Albuquerque, C. P., Fonseca, A. C., Simões, M. R., Pereira, M., Rebelo, J. A., \& Temudo, P. (1998). O Inventário de Comportamento da Criança para Pais (I. C. C. P.). In M. R. Simões, M. M. Gonçalves, \& L. S. Almeida (Eds.), Testes e provas psicológicas em Portugal (vol. II, pp. 21-36). Braga: APPORT/ SHO.

Azevedo, M.A., \& Guerra, V. N. A. (1989). Vitimação e vitimização: Questões conceituais. In M. A. Azevedo \& V. N. A. Guerra (Eds.), Crianças vitimizadas: A síndrome do pequeno poder (pp. 43-47). São Paulo: Iglu Editora.

Bernard, H., Killworth P., Kronenfeld D., \& Sailor L. (1984). The problem of informant accuracy: The validity of retrospective data. Annual Review of Anthropology, 13, 495-517. doi: 10.1146/annurev.an.13.100184.002431

Beesdo, K., Knappe, S., \& Pine, D. S. (2009). Anxiety and anxiety disorders in children and adolescents: Developmental issues and implications for DSM-V. Psychiatric Clinics of North America, 32(3), 483-524. doi: 10.1016/j.psc.2009.06.002

Bohanek, J. G., Fivush, R., \& Walker, E. (2005). Memories of positive and negative emotional events. Applied Cognitive Psychology, 19, 51-66. doi: 10.1002/acp.1064

Bower, G. H. (1981). Mood and memory. American Psychologist, 36, 129-148. doi: 10.1037/0003-066X.36.2.129 
Brewer, W. F. (1994). Autobiographical memory and survey research. In N. Schwarz \& S. Sudman (Eds.), Autobiographical memory and the validity of retrospective reports (pp. 11-20). New York: Springer-Verlag. doi: 10.1007/978-1-4612-2624$6 \_2$

British Psychological Society. (2008). Guidelines on memory and the law: Recommendations from the scientific study of human memory. Recuperado de http://www.policecouncil.ca/reports/ BPS\%20Guidelines\%20on\%20Memory.pdf

Brown, M. (2012). Assessing recall of early life circumstances: Evidence from the National Child Development Study (Working Paper). Centre for Longitudinal Studies, Institute of Education, University of London.

Conway, M. A. (2005). Memory and the self. Journal of Memory and Language, 53, 594-628. doi: 10.1016/j.jml.2005.08.005

Conway, M. A., \& Pleydell-Pearce, C. W. (2000). The construction of autobiographical memories in the self-memory system. Psychological Review, 107, 261-288. doi: 10.1037/0033295X.107.2.261

Coolidge, F., Tambone, G., Durham, R., \& Segal, D. (2011). Retrospective assessments of childhood psychopathology by adults and their parents. Psychology, 2, 162-168. doi: 10.4236/ psych.2011.23026

Ensminger, M. E., Juon, H. S., \& Green, K. M. (2007). Consistency between adolescent reports and adult retrospective reports of adolescent marijuana use: Explanations of inconsistent reporting among an African American population. Drug and Alcohol Dependence, 89, 13-23. doi: 10.1016/j. drugalcdep.2006.11.011

Fisher, H. L., Bunn, A., Jaccobs, C., Moran, P. \& Bifulco, A. (2011). Concordance between mother and offspring retrospective reports of childhood adversity. Child Abuse Neglect, 35(2), 117-122. doi: 10.1016/j.chiabu.2010.10.003

Fonseca, A. C., \& Perrin, S. (2011). The clinical phenomenology and classification of child and adolescent anxiety. In W. K. Silverman \& A. P. Field (Eds.), Anxiety disorders in children and adolescents (2nd ed., pp. 25-55). Cambridge: Cambridge University Press.

Fonseca, A. C., Simões, A., Rebelo, J. A., Ferreira, J. A. A., \& Cardoso, F. (1994). Um inventário de competências sociais e de problemas de comportamento em crianças e adolescentes: O Child Behaviour Checklist de Achenbach (CBCL). Psychologica, 12, 55-78.

Fonseca, A. C., Simões, A., Rebelo, J. A., Ferreira, J. A. A., \& Cardoso, F. (1995). O Inventário de Comportamento da Criança para Professores: Teachers Report Form (TRF). Revista Portuguesa de Pedagogia, 29(2), 81-102.

Greenhoot, A. F. (2011). Retrospective methods in developmental science. In B. Laursen, T. Little, \& N. Card (Eds.), Handbook of developmental research methods (pp. 196-210). New York, NY: Guilford.

Grysman, A., \& Hudson, J. A. (2013). Gender differences in autobiographical memory: Developmental and methodological considerations. Developmental Review, 33(3), 239-272. doi: 10.1016/j.dr.2013.07.004

Hardt, J., \& Rutter, M. (2004). Validity of adult retrospective reports of adverse childhood experiences: Review of the evidence. Journal of Child Psychology and Psychiatry, 45(2), 260-273. doi: 10.1111/j.1469-7610.2004.00218.x
Hardt, J., Sidor A, Bracko M., \& Egle, U. T. (2006). Reliability of retrospective assessments of childhood experiences in Germany. Journal of Nervous and Mental Disease, 194(9), 676-683. doi: 10.1097/01.nmd.0000235789.79491.1b

Loftus, E. F. (1975). Leading questions and the eyewitness report. Cognitive Psychology, 7, 560-572. doi: 10.1016/00100285(75)90023-7

Loftus, E. F., \& Cahill, L. (2007). Memory distortion: From misinformation to rich false memory. In J. S. Nairne (Ed.), The foundations of remembering: Essays in honor of Henry L. Roediger III (pp. 413-425). Hove: Psychology Press.

McDougall, P., \& Vaillancourt, T. (2015). Long-term adult outcomes of peer victimization in childhood and adolescence pathways to adjustment and maladjustment. American Psychologist, 70(4), 300 -310. doi: 10.1037/a0039174

McLean, K. C., Pasupathi, M., \& Pals, J. L. (2007). Selves creating stories creating selves: A process model of self-development. Personality and Social Psychology Review, 11(3), 262-278. doi: 10.1177/1088868307301034

Murphy, D. M. (1985). Fears in preschool-age children. Child Care Quarterly, 14(3), 171-189. doi: 10.1007/BF01113746

Neisser, U. (1994). Self narratives: True and false. In U. Neisser $\&$ R. Fivush (Eds.), The remembering self: Construction and accuracy in the self-narrative (pp. 1-18). New York: Cambridge University Press.

Offer, D., Kaiz, M., Howard, K. J., \& Bennett, E. S. (2000). The altering of reported experiences. Journal of the American Academy of Child \& Adolescent Psychiatry, 39(6), 735-742. doi: 10.1097/00004583-200006000-00012

Pasupathi, M., \& Wainryb, C. (2010). On telling the whole story: Facts and interpretations in autobiographical memory narratives from childhood through mid-adolescence. Developmental Psychology, 46(3), 735-746. doi: 10.1037/a0018897

Picard, L., Eustache, F., \& Piolino, P. (2009). De la mémoire épisodique à la mémoire autobiographique: Approche développementale. L'Année Psychologique, 9, 197-236. doi: 10.4074/S0003503309002012

Pillemer, D. B., \& White, S. H. (1989). Childhood events recalled by children and adults. In H. W. Reese (Ed.), Advances in child development and behavior (vol. 21, pp. 297-340). New York: Academic Press. doi: 10.1016/S0065-2407(08)60291-8

Rebelo, J. A., Fonseca, A. C., Simões, A., \& Ferreira, J. A. (1995). Dificuldades de aprendizagem: Elementos para a caracterização do problema. Revista Portuguesa de Pedagogia, XXIX(3), 35-54.

Renk, K., Roberts, R., Klein, J., Rojas-Vilches, A., \& Sieger, K. (2005). Retrospective reports of college students' childhood problems. Journal of Clinical Psychology, 61(3), 235-250. doi: 10.1002/jclp. 20018

Ritchie, T. D., Batteson, T. J., Bohn, A., Crawford, M. T., Ferguson, G. V., Schrauf, R. W., Vogl, R. J., \& Walker, W. R. (in press). A pancultural perspective on the fading affect bias in autobiographical memory. Memory. doi: $10.1080 / 09658211.2014 .884138$

Ros, L., \& Latorre, J. M. (2010). Gender and age differences in the recall of affective autobiographical memories using the autobiographical memory test. Personality and Individual Differences, 49, 950-954. doi: 10.1016/j.paid.2010.08.002 
Ross, M., \& Buehler, R. (1994). On authenticating and using personal recollections. In N. Schwarz \& S. Sudman (Eds.), Autobiographical memory and the validity of retrospective reports (pp. 55-69). New York: Springer-Verlag. doi: 10.1007/978-1-4612-2624-6_5

Schwartz, N., \& Sudman, S. (1994). Introduction and overview. In N. Schwarz \& S. Sudman (Eds.), Autobiographical memory and the validity of retrospective reports (pp. 1-8). New York: Springer-Verlag. doi: 10.1017/CBO9780511811333.002

Sullivan, G. M. (2011). A primer on the validity of assessment instruments. Journal of Graduate Medical Education, 3(2), 119-120. doi: 10.4300/JGME-D-11-00075.1

Wagenaar, W. A. (1986). My memory: A study of autobiographical memory over six years. Cognitive Psychology, 18, 225-252. doi: 10.1016/0010-0285(86)90013-7
Wang, Q., Leichtman, M. D., \& White, S. H. (1998). Childhood memory and self-description in young Chinese adults: The impact of growing up an only child. Cognition, 69(1), 73-103. doi: 10.1016/S0010-0277(98)00061-4

Wells, C., Morrison, C. M., \& Conway, M. A. (2013). Adult recollections of childhood memories: What details can be recalled? The Quarterly Journal of Experimental Psychology, 67(7), 1249-1261. doi: 10.1080/17470218.2013.856451

Westbury, C., \& Dennett, D. C. (2000). Mining the past to construct future: Memory and belief as forms of knowledge. In D. L. Schacter \& E. Scarry (Eds.), Memory, brain, and belief (pp. 11-32). Cambridge, MA: Harvard University Press.

White, H. R., Widom, C. S., \& Chen, P-H. (2007). Congruence between adolescents' self-reports and their adult retrospective reports regarding parental discipline practices during adolescence. Psychological Reports, 101, 1079-1094. doi: 10.2466/pr0.101.4.1079-1094

Recebido em 05.08.2014

Primeira decisão editorial em 13.06.2016

Versão final em 28.07.2016

Aceito em 22.08.2016 